\title{
Accesibilidad física de la población a servicios de salud pública en San Pelayo y Cereté, Córdoba, Colombia, año 2015
}

PHYSICAL ACCESSIBILITY OF THE POPULATION TO PUBLIC HEALTH SERVICES IN SAN PELAYO AND CERETÉ, CÓRDOBA, COLOMBIA, YEAR 2015

\section{ACESSIBILIDADE FÍSICA DA POPULAÇÃO AOS SERVIC̣OS DE SAÚDE PÚBLICA EM SAN PELAYO E CERETÉ, CÓRDOBA, COLÔMBIA, ANO 2015}

Para citar este artículo: Hernández Gene, F. J. y Garnica Berrocal, R. (2017). Accesibilidad física de la población a servicios de salud pública en San Pelayo y Cereté, Córdoba, Colombia, año 2015. Perspectiva Geográfica, 22(2), 67-84. doi: $10.19053 / 01233769.5956$
FRANCISCO JAVIER Hernández Gene ${ }^{1}$ ROSANA GARNICA Berrocal²
Recepción:

3 de abril de 2017

Evaluación:

31 de octubre de 2017

Aprobación:

16 de noviembre de 2017

\section{Resumen}

Este artículo analiza la accesibilidad física de la población a servicios de salud pública, como los Centros de Salud, los Centros de Atención Médica Urbana (CAMU), ambos

1 Geógrafo de la Universidad de Córdoba. Miembro del grupo de investigación Estudios Urbano-Regionales del Caribe Colombiano. geojavier.11@gmail.com

2 Arquitecta de la Universidad Santo Tomás de Aquino (seccional Bucaramanga, Colombia), especialista en Administración y Planificación del Desarrollo Regional con énfasis en Ordenamiento Territorial (Universidad de Los Andes, Colombia) y Magíster en Geografía (Universidad de Córdoba, Colombia). Docente de planta de la Universidad de Córdoba, programa de Pregrado en Geografía.rgarnica@correo.unicordoab.edu.co,garna79@gmail.com 
de I nivel de atención, y el Hospital (II nivel), en las municipalidades de San Pelayo y Cereté, Colombia. Las variables físico-espaciales, cualitativas (medio de transporte, tipo de vía) y cuantitativas (velocidades según tipo de vía, número de habitantes, tiempos de recorrido) determinan las categorías de accesibilidad física según el tiempo de recorrido (óptima, favorable, desfavorable, muy desfavorable) para cada tipo de equipamiento. En el occidente del territorio, el relieve acolinado es desfavorable para acceder a los servicios de salud, por las largas distancias de recorrido y en el oriente, la zona plana (planicie aluvial del río Sinú) y la accesibilidad es favorable y óptima. El 80\% de la población se moviliza en motocicleta debido a la ausencia de transporte público y a la existencia de vías sin pavimentar $(93,7 \%)$ cuyas velocidades no superan los $40 \mathrm{~km} / \mathrm{h}$.

Palabras clave: accesibilidad fisica, Cereté, Colombia, desigualdad social, población, San Pelayo, servicios de salud pública.

\section{Abstract}

This article analyzes the physical accessibility of the population to public health services, such as Health Centers, the Urban Health Care Centers (CAMU), Hospital), both at the I level of care, and the Hospital (II level) in municipalities of San Pelayo and Cereté (Colombia). The physical-spatial, qualitative variables (means of transport, type of track) and cuantitatives variables (velocities according to road type, number of inhabitants, travel times) determine physical accessibility categories through travel time (optimal, favorable, unfavorable, very unfavorable) for each type of equipment. In the west of the territory, the relief is unfavorable to access the health services, for the long distances of travel and the east, the flat area (alluvial plain of the river Sinu) accessibility is favorable and optimal. The principal conveyance is motorcycle (80\%) since public transport not exist either pavement roads $(93,7 \%)$; the speed don't exceed $40 \mathrm{~km}-\mathrm{h}$.

Keywords: Physical accessibility, Cereté, San Pelayo, Colombia, Public health services, social inequality, population.

\section{Resumo}

Este artigo analisa a acessibilidade física da população aos serviços de saúde pública, como os Postos de Saúde, os Centros de Atenção Médica Urbana (CAMU), ambos de I nível de atenção, e o Hospital (II nível), nas municipalidades de San Pelayo e 
Cereté, Colômbia. As variáveis físico-espaciais, qualitativas (médio de transporte, tipo de via), e quantitativas (velocidades segundo tipo de via, número de habitantes, tempos de recorrido), determinam as categorias de acessibilidade física segundo o tempo de recorrido (ótima, favorável, desfavorável, muito desfavorável) para cada tipo de equipamento. No oeste do território, o relevo colinoso é desfavorável para acessar aos serviços de saúde pelas longas distâncias de recorrido, e no leste, a zona plana (planície aluvial do rio Sinú) a acessibilidade é favorável e ótima. O 80\% da população mobiliza se de motocicleta devido à ausência de transporte público e à existência de vias sem asfaltar (93,7\%) cujas velocidades não superam os $40 \mathrm{~km} / \mathrm{h}$.

Palavras chave: acessibilidade fisica, Cereté, Colômbia, desigualdade social, população, San Pelayo, serviços de saúde pública.

\section{Introducción}

En Colombia, la Constitución Política de 1991 en su artículo 49 y la Ley 1751 del 17 de febrero de 2015, aprobada por el Congreso de la República, mediante la cual se regula el derecho fundamental a la salud y se dictan otras disposiciones, define la salud como un servicio público a cargo del Estado, de carácter obligatorio, en el que se garantiza a todas las personas el acceso a los servicios de promoción, protección y recuperación de la salud y el acceso a la igualdad en términos de la accesibilidad (económica y física), entre otros, como elemento y principio del derecho fundamental a la salud (Artículo 6 de la Ley 1751).

Los servicios de salud son de gran importancia y juegan un papel fundamental en la sociedad, ya que de estos depende el desarrollo, bienestar y la calidad de vida de las personas que habitan un territorio, por lo cual la accesibilidad geográfica a estos servicios, en específico a Centros de Salud, Centros de Atención
Médica Urbana (en adelante CAMU) ${ }^{3}$, clasificados en el primer nivel de complejidad y Hospitales en el segundo nivel de atención, como lo reglamenta el Ministerio de Salud de la República de Colombia en la Resolución número 5261 de 1994, es una obligación. Ello implica la existencia misma de los servicios y la facilidad de la población para acceder a estos en términos físicos (accesibilidad física) y económicos (accesibilidad socioeconómica). Estos criterios en su conjunto conforman la definición de la accesibilidad geográfica, según lo definen Escalona y Diez (2002).

Es de anotar que la accesibilidad geográfica a servicios de salud es un tema tratado a nivel mundial, regional y nacional, sobresaliendo en España autores como Escalona y Diez (2002) y Rodríguez (2010); en Ruanda (África), Huerta y Källestål (2012); en

3 Los Centros de Salud son ambulatorios (consulta médica general y/o personal auxiliar, y otros profesionales de la salud), mientras que en los CAMU la atención es ambulatoria especializada en urgencias, medicina especializada en ginecología y obstetricia, sin ser quirúrgica. A diferencia de los anteriores, el Hospital cuenta con urgencias, hospitalización, cirugía, apoyo diagnóstico y terapéutico. 
Argentina, Ramírez (2009) y Villanueba (2010), y en Colombia, Pérez (2013), entre otros. Ellos tienen en cuenta las distancias empleadas bien sea en longitud (metros) o tiempo (minutos), según el tipo de vía y medio de transporte utilizado, relacionados con la cantidad de población por área que accede a los equipamientos de salud.

Por su parte, la accesibilidad física, la cual es objeto central de este artículo, se refiere a la existencia del servicio y a los medios para llegar hasta este. Igualmente, se entiende como accesibilidad potencial, estudiada a través de variables relativas a la localización de centroides (centro geométrico de los corregimientos y las áreas urbanas), el número de habitante de tales áreas, su distancia a la demanda del servicio como son centros de salud, CAMU y Hospital (Joseph y Phillips citado por Escalona y Díez, 2002).

A pesar de no ser abordada la accesibilidad socioeconómica en este artículo, esta se trata de la capacidad que tiene la población para usar tal servicio, permite valorar la calidad de este (buena o mala), de acuerdo con los horarios de atención, tiempo de espera para ser atendido y condiciones que regulan su funcionamiento, por ejemplo, días a la semana en ofrecer atención a los usuarios, etc.

En este sentido, en Colombia y particularmente en las municipalidades de Cereté y San Pelayo, existen desigualdades e inequidades en términos de accesibilidad física hacia los servicios de salud pública, ya que las personas utilizan los servicios de manera diferencial, bien sea por la existencia de grandes diferencias en términos de distancias entre unos servicios y otros (centros de salud, CAMU, Hospital), influenciadas por los tipos de vías por las cuales transitan, generando largos y cortos tiempos de recorrido en unas áreas que comparadas entre sí registran valores distintos (desiguales) por las velocidades permitidas a lo largo de su circulación, en asociación con el medio de transporte utilizado.

El objetivo del artículo es analizar la accesibilidad física de la población a los servicios de salud pública ofertados en el área de estudio para explicar las condiciones físicas mediante las cuales la población accede a estos, a través de la medición de variables físico-espaciales. Para ello se inicia con la definición del medio de transporte más utilizado por la población para dirigirse a los servicios de salud, posteriormente se realiza una clasificación vial, se determinan las velocidades a las cuales se puede circular dependiendo del tipo de vía, se operacionalizan los indicadores tiempos de recorridos (isócronas hacia cada tipo de servicios de salud pública), número de personas por unidad espacial en el área de estudio, teniendo en cuenta la localización de los equipamientos de salud pública y los centros poblados (división político-administrativa rural de los municipios en Colombia). A partir de lo anterior, se determinan las categorías de accesibilidad física (óptima, favorable, desfavorable, muy desfavorable) para cada tipo de equipamiento con base en Escalona y Díez (2002).

Esta investigación es abordada desde la Geografía de la Salud mediante el uso del enfoque de localización de los servicios, el cual se originó con la institucionalización del paradigma cuantitativo de fuerte influencia positivista en la comunidad geográfica, durante la década de los cincuenta, teniendo gran receptividad por geógrafos que se interesan por el equipamiento sanitario, su distribución y accesibilidad geográfica, etc. (Borroto, Somarribas y Chamizo, 1994). Así, el presente trabajo constituye una línea base para la planificación del desarrollo urbano y territorial, las cuales son de competencia 
de las entidades privadas y públicas de nivel local, departamental y nacional (promotoras de salud, alcaldías, gobernación, secretarías de salud, Ministerio de la Protección Social, entre otras), buscando mejorar la calidad de vida de la sociedad a partir de la comprensión de la importancia que adquieren los servicios de salud pública y su relación con la población en cuanto a su accesibilidad física.

\section{2. Área de estudio}

Los municipios de San Pelayo y Cereté están localizados al norte de Colombia en la parte noroccidente del departamento de Córdoba en una extensión aproximada de 72715 hectáreas, limitando al norte con los municipios de Lorica, Cotorra, Chimá; al oriente con Ciénaga de Oro, al extremo sur con Montería, San Carlos y hacia el occidente con Puerto Escondido (Figura 1).

Las unidades espaciales son los 21 corregimientos (áreas rurales) que conforman el área de estudio, distribuidos en 12 que pertenecen al municipio de San Pelayo, como son: Bongamella, Buenos Aires,
Caño Viejo Valparaíso, Carrillo, El Chiquí, El Obligado, La Madera, Las Guamas, Pelayito, Puerto Nuevo, Sabana Nueva, San Isidro, y 9 restantes de Cereté, Cuero Curtido, Manguelito, Martínez, Mateo Gómez, Rabolargo, Retiro de Los Indios, Severá, Tres María, Venado Campanito; además de las dos áreas urbanas o cabeceras municipales de estos dos municipios.

El territorio estudiado consta, por una parte, de una zona plana en la planicie aluvial del río Sinú, una de las principales cuencas hidrográficas de Colombia, el cual atraviesa de sur a norte a varios municipios del departamento de Córdoba. Presenta alturas entre los 12 y $30 \mathrm{~m}$ s.n. m., la conforman los corregimientos y cabeceras municipales del norte y suroriente de San Pelayo y Cereté. Por otra parte, de la topografía ondulada y semiondulada de la serranía de Abibe, con alturas entre 30 m s.n.m. y 120 m s. n. m., se observan al occidente, a excepción de las superiores a 120 metros, con un máximo de 240 metros, en algunos corregimientos donde se destacan formaciones de lomas, cerro y cuchillas.
FIGURA 1.

Localización de los municipios de San Pelayo y Cereté en el departamento de Córdoba, Colombia

Fuente: Elaboración propia.

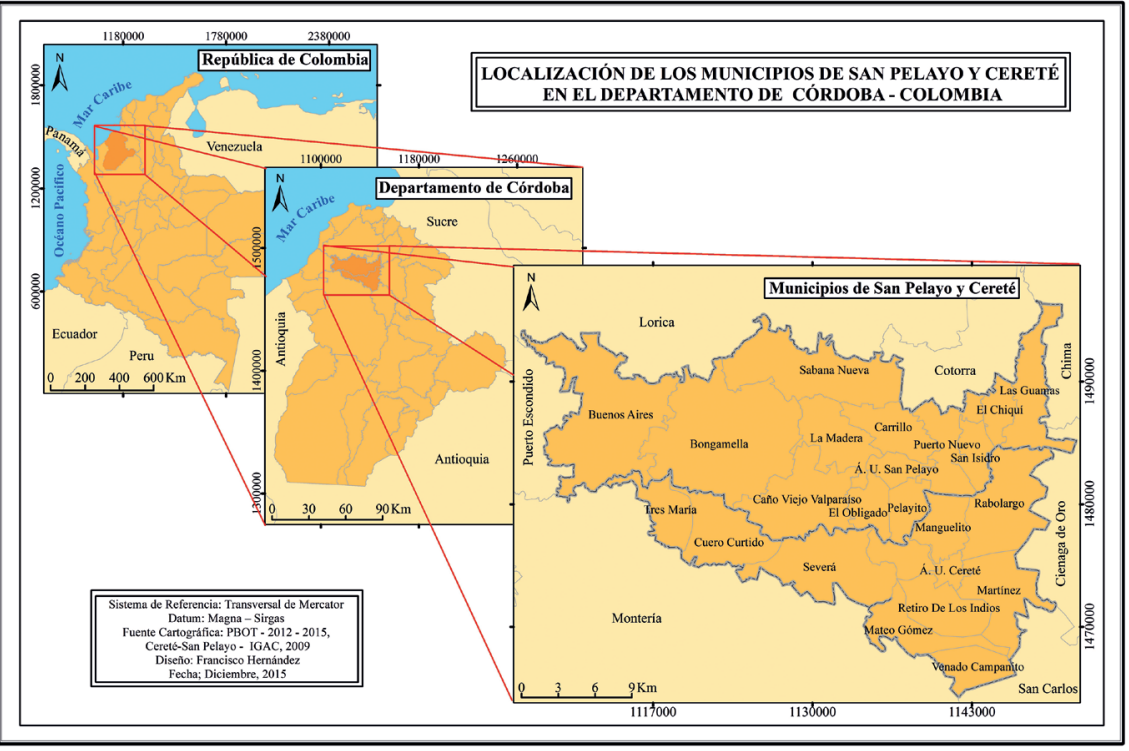




\section{Metodología}

El método de investigación empleado es mixto puesto que aborda variables físico-espaciales cuantitativas y cualitativas, las primeras referidas a las velocidades según tipo de vía, número de habitantes y los tiempos de recorrido, siendo estos últimos determinantes en las categorías de accesibilidad física (óptima, favorable, desfavorable, muy desfavorable), y las segundas (clasificación vial y de los medios de transporte). La investigación es de tipo analítico, debido a que analiza la accesibilidad física de la población a los servicios de salud pública en los municipios de San Pelayo y Cereté (Córdoba), para comprender las condiciones físicas que posibilitan o no el acceso de la población a estos.

Por un lado, en la recolección de la información primaria se aplicó una encuesta para indagar el medio de transporte utilizado por la población, el tipo de servicio recurrido, la influencia de la época de precipitaciones en el acceso a los servicios de salud en las diferentes corregimientos y áreas municipales, teniendo presente su total en el área de estudio (128460 habitantes), según fuente oficial del Sistema de Identificación de Potenciales Bene- ficiarios de Programas Sociales (SISBEN, 2015), el cual depende de las alcaldías municipales. Se diseñó un muestreo estratificado aleatorio simple con un margen de error del $4 \%$ cuyo resultado arrojó una muestra de 597 personas, quienes fueron distribuidas en las diferentes unidades espaciales (corregimientos y áreas urbanas). La localización de los servicios de salud se obtuvo en campo mediante el sistema de posicionamiento global (GPS).

Por otro lado, en la recolección de información secundaria se obtuvo la clasificación vial según el tipo de vía y las velocidades a las cuales las personas pueden transitar en vehículos automotores teniendo en cuenta los medios de transporte (Instituto Geográfico Agustín Codazzi, 2009; Ministerio de Transporte de la República de Colombia, 2002, 2010) Tabla 1.

Teniendo la información recolectada, se procede a su organización y clasificación en una geodatabase (Figura 2) y con la ayuda de los Sistemas de Información Geográfica (SIG), a través del software ArcGis 10.3, se calcula la accesibilidad física según el tiempo de recorrido, siguiendo la metodología realizada por Escalona y Díez (2003).

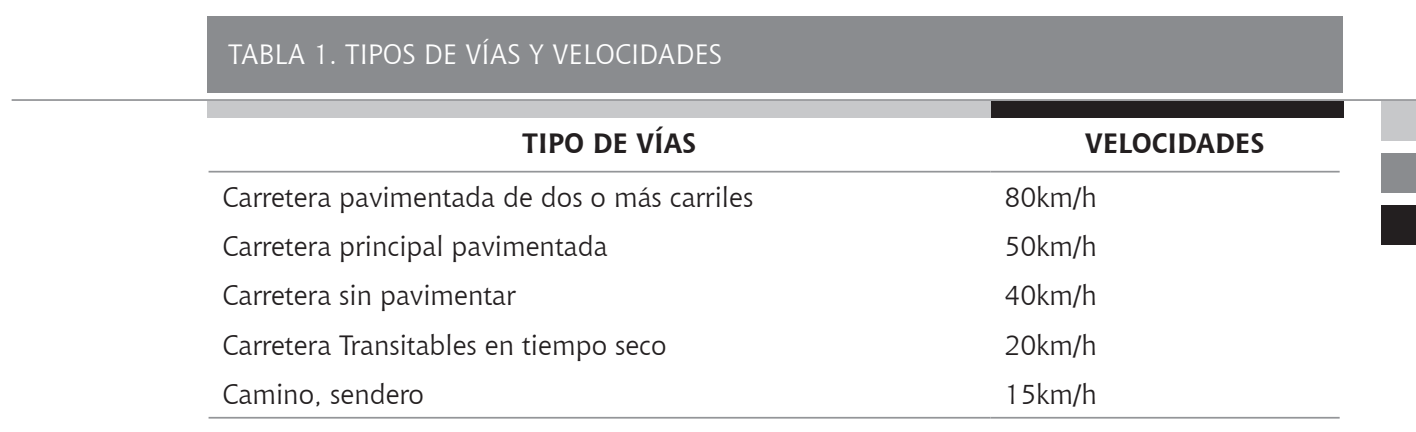

Fuente: Elaboración propia con base en IGAC (2009), Ley 769 de 2002, Resolución 1384 de 2010. 


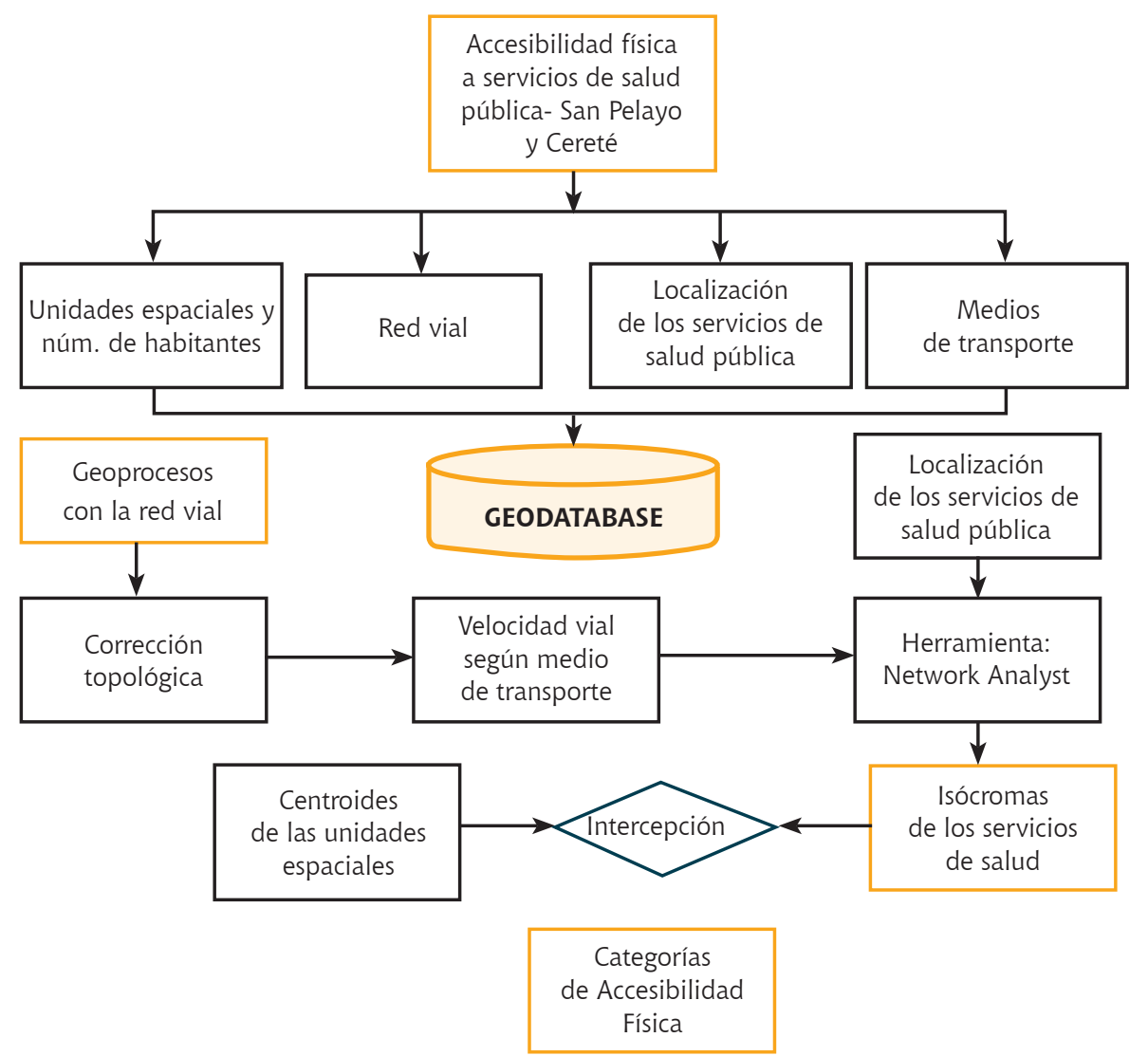

FIGURA 2.

Esquema metodológico en la obtención de la accesibilidad física a los servicios de salud pública Fuente: Elaboración propia.
La fórmula de la accesibilidad física empleada es $A_{i}=g\left(W_{j}\right) f\left(c_{i j}\right)$, con $W_{j}=1$

Donde $\mathrm{A}_{\mathrm{i}}$ es la accesibilidad potencial agregada del lugar $\mathrm{i}, \mathrm{W}_{\mathrm{j}}$ es la actividad o servicio $\mathrm{W}$ que se ofrece en $\mathrm{j}, \mathrm{y}_{\mathrm{ij}}$ es el coste general de llegar a j desde i. Es usual llamar a las funciones $\mathrm{g}\left(\mathrm{W}_{\mathrm{j}}\right)$ y $\mathrm{f}\left(\mathrm{c}_{\mathrm{ij}}\right)$ funciones de actividad y funciones de impedancia, respectivamente. Por su parte, la función de actividad es horizontal y expresa que solo hay un destino posible (al que se le da valor 1) y, por otra parte, la función de impedancia es lineal o dependiente únicamente del coste de desplazamiento. En consecuencia, $\mathbf{A}_{\mathrm{i}}$ es solo función de $\mathbf{c}_{\mathrm{ij}}$, $\mathrm{y}$ de hecho toma su valor. El resultado expresa una desutilidad, es decir, cuanto más bajo es dicho valor mayor es la accesibilidad. Los resultados son analizados según criterios de accesibilidad física en el que se tienen en cuenta el tiempo de recorrido en minutos hacia cada tipo de servicio de salud pública (centros de salud, CAMU y Hospital), así:

\begin{tabular}{lll}
\multicolumn{2}{l}{ TABLA 2. CATEGORÍAS DE ACCESIBILIDAD FISICA SEGÚN EL TIEMPO DE RECORRIDO EN } \\
MINUTOS & \multicolumn{1}{c}{ TIEMPO DE ACCESO EN MINUTOS } & \multicolumn{1}{c}{ CATEGORÍA DE ACCESIBILIDAD FÍSICA } \\
\hline$A_{i}=f\left(c_{i j}\right)$, con $c_{i j} \leq 15$ minutos & Accesibilidad óptima \\
$A_{i}=f\left(c_{i j}\right)$, con $15<c_{i j} \leq 30$ minutos & Accesibilidad favorable/aceptable \\
$A_{i}=f\left(c_{i j}\right)$, con $30<c_{i j} \leq 45$ minutos & Accesibilidad desfavorable \\
$A_{i}=f\left(c_{i j}\right)$, con $c_{i j}>45$ minutos & Accesibilidad muy desfavorable \\
\hline
\end{tabular}

Fuente: Elaboración propia con base en Escalona y Díez (2003). 
Finalmente, se realizó el análisis de los resultados partiendo de la idea de que las condiciones de accesibilidad física de la población a los servicios de salud pública en los municipios de San Pelayo y Cereté (Córdoba) guardan relación con las condiciones físicas que posibilitan o no el acceso de la población a estos.

\section{Resultados y discusión}

El acceso a los servicios de salud se relaciona tanto con la presencia del equipamiento como con su alcance geográfica y económicamente, en lo que el transporte público adquiere gran importancia por su influencia central en dicho acceso, representando obstáculos a superar (Villanueba, 2010) u oportunidades que aprovechar. En términos cuantitativos, Fuenzalida (2010) señala que la ciencia geográfica emplea índices de accesibilidad para evaluar las condiciones existentes y futuras de eficiencia o equidad, teniendo en cuenta que la eficiencia se valora en la medida que la distribución de la oferta (los recursos) posibilite el alcance máximo de accesibilidad espacial. En este sentido, Gutiérrez y García (2002) enfatizan en dos indicadores muy usuales para medir la accesibilidad física: la separación geográfica (distancias métricas) y los de espacio-tiempo (modos de transporte y tiempos empleados en los trayectos).

Al respecto, el estudio de la accesibilidad física de la población a servicios de salud pública en San Pelayo y Cereté (Colombia) al año 2015 se hizo a partir de los tiempos de recorrido en torno a la incidencia que tienen el estado y el tipo de las vías, permitiendo la circulación a mayor o menor velocidad según el tipo de transporte utilizado por ella en el territorio, (Villanueba, 2010), lo que trae consigo desigualdades territoriales. Esto permite sintetizar las oportunidades de contacto entre la población y las infraestructuras de salud. Es decir, que ella buscaría reducir las distancias lineales, usando los servicios más cercanos.

En este orden de ideas, en el área de estudio se pudo identificar la población asentada en las unidades espaciales que se encuentran en las mejores y peores condiciones de accesibilidad a los servicios de salud pública, que en el último caso, se presenta tras no haber atención médica en temporadas de lluvias, como sucede en los corregimientos de Buenos Aires, Sabana Nueva, Bongamella, Las Guamas, Severá, Cuero Curtido y Tres María, viéndose más afectado el municipio de Cereté. Esto resulta relevante por aportar información y datos sobre la accesibilidad física de la población como parte de la situación actual del equipamiento de salud, pues las dos municipalidades carecen de este tipo de estudios.

La interpretación de los resultados permite analizar que en los municipios de San Pelayo y Cereté se evidencian desigualdades e inequidades manifestadas por los niveles diferenciales de la accesibilidad física de la población a los servicios de salud. En palabras de Villanueba (2010), esta situación refleja un elemento más de fragmentación dentro de las ciudades así como entre ellas, según el estudio similar que realizó sobre los sistemas de salud y educación para las localidades de Necochea y Quequén en Argentina.

Particularmente en San Pelayo y Cereté, estas desigualdades e inequidades son manifestadas en mayor medida por los usuarios rurales que acceden óptimamente a las infraestructuras de salud con el menor nivel de atención I (Centros de Salud y CAMU) a excepción de Tres María, Pelayito y el área urbana de San Pelayo, limitándose el acceso a la oferta de servicios con mayor complejidad, si se comparan con aquellos de la cabecera municipal 
quienes lo hacen de manera diferenciada, gozando de mejores condiciones en la prestación de estos, como sucede con el Hospital Regional de Cereté (II nivel de atención), localizado en el área urbana, el cual ofrece mayor complejidad en los servicios ofertados a una población urbana pese a que el predominio a nivel municipal es rural en el caso de San Pelayo.

Los centros de salud prestan los servicios algunos días de la semana debido a la disponibilidad de personal médico, quienes laboran entre tres y cinco, y solo en la jornada diurna, entre los que caben mencionar: medicina general, odontología, laboratorio clínico y programas de control prenatal, de crecimiento y desarrollo en el menor de 5 años, planificación familiar, prevención y control de enfermedades diarreicas, respiratorias, prevención y control de hipertensión arterial, inmunización (aplicación de vacunas), saneamiento básico ambiental, control de zoonosis, de establecimientos públicos, atención de enfermería, remisión a consulta especializada.

Es de considerar que las infraestructuras físicas como las viales y el transporte limitan el acceso de la población a los equipamientos de salud, puesto que inciden en el tiempo que tardan para llegar a estos, lo que se convierte en una problemática o deficiencia que deberán solucionar las administraciones municipales en aras de garantizar el acceso a la igualdad en términos de la accesibilidad física, como lo plantea el Congreso de la República de Colombia (1991). Sin embargo, en la realidad nacional, particularmente la municipal (urbana y rural) no se demuestran tales garantías; sí se observan, por el contrario, una accesibilidad desfavorable y muy desfavorable del área de estudio, la cual está asociada al mal estado vial, principalmente sin pavimentar y a las vías tipo caminos, senderos, transitables en tiempo seco, y cuyas velocidades máximas son de $40 \mathrm{~km} / \mathrm{h}$.
Además, el uso generalizado del transporte informal (motocicleta) por parte de la población al momento de dirigirse hacia estos equipamientos es de manera obligatoria debido a la ausencia de transporte público que posibilite su movilidad de forma eficiente, segura, a bajo costo y de buena calidad, en atención a las necesidades de los usuarios, en articulación con la adecuada red vial. Pareciera contradictorio el hecho de tener población asentada en las proximidades a los centros de salud, quien manifestó en las encuestas dirigirse en mayor medida a un CAMU y al Hospital, a pesar de haber solo tres equipamientos de salud pública oficial concentrados en el área urbana.

La situación antes descrita no necesariamente conduce a pensar de manera restringida en el tema de la movilidad y el transporte, sino en las implicaciones que esta trae en términos de la competitividad y la articulación funcional y territorial (atributos sociales, económicos, culturales, políticos), en lo que a la eficiencia, la calidad y la identidad territorial se refieren, en busca de lograr territorios cohesionados con desarrollo equilibrado, justo y equitativo, tendiendo a disminuir las brechas entre los espacios rurales y urbanos, las regiones y los departamentos, lo que se convierte en un reto y desafío para la planificación territorial en América Latina y El Caribe, la cual poco ha sido desarrollada, al revisar los indicadores de desigualdad (pobreza, Gini, empleo, acceso a los servicios de educación y salud, entre otros).

\subsection{Los medios de transporte y la red vial a través de los cuales la población accede a los servicios de salud pública}

Los municipios de San Pelayo y Cereté concentran 128460 habitantes, en un área de 72715 ha, distribuidas heterogéneamente entre los corregimientos 
y las zonas urbanas, ocupando solo el $2 \%$ de cada uno en su perímetro urbano con 918 ha y 570 ha, respectivamente), mientras que el $98 \%$ restante es rural (44068 y 27184 ha).

Particularmente en Cereté, con respecto a la población total de 85389 habitantes, el 56\% se localiza en la cabecera municipal (47987), mientras que el $44 \%$ (37402) restante vive en el área rural, en comparación con San Pelayo que cuenta con menor población, teniendo un total de 43071 , siendo más rural el $82 \%$ de su población con respecto al total (35270) y únicamente el 18\% es urbana (7801).

Estos asentamientos emplean varios medios de transporte para desplazarse hacia los servicios de salud pública, tales como la motocicleta, el vehículo automotor particular, de servicio público informal y formal, a pie, la bicicleta o la combinación de varios de estos. Al respecto, la motocicleta, predomina sobre los demás teniendo en cuenta que un $80 \%$ (480 personas encuestadas) del total de la muestra (597) la usan antes que cualquier otro; y el $20 \%$ restante se distribuye en varios medios, correspondiendo el $9 \%$ a 49 personas, otros (a pie, bicicleta) al 6\% (34 personas), los vehículos automotores particular e informal $2 \%$ cada uno (total 28 personas) y el vehículo público formal el $1 \%$ (6 personas).

Es así que parte de las debilidades se presentan en los territorios colombianos indistintamente de ser urbanos o rurales en cuanto a la disponibilidad de la oferta que demandan los pobladores sobre el uso de transporte público, la cual no es cubierta hecho que lleva a que ellos se movilicen en otros medios, adaptándose en gran medida a las condiciones de la red vial, mayormente destapada $(93,7 \%)$ y distribuida en caminos, senderos (46,7\%), carreteras transitables en tiempo seco $(25,2 \%)$, carretera sin pavimentar (21,8\%); y en menor proporción, el 6,3\% restante, lo constituyen las carreteras pavimentadas de dos o más carriles.

Teniendo en cuenta lo anterior, las vías son intransitables ( $\sin$ pavimento) en época de precipitaciones, ya que empeoran su mal estado, limitando el acceso de los usuarios a los equipamientos de salud pública, como sucede en los corregimientos Buenos Aires, Sabana Nueva, Bongamella, Las Guamas, Severá. Cuero Curtido y Tres María, donde no hay atención médica en esta época. Estos corregimientos coinciden con estar dentro de las categorías de accesibilidad física desfavorable o muy desfavorable, lo que indica la necesidad de mejorar las condiciones de la red vial, para así propiciar espacios de fácil movilidad de un lugar a otro desde sus residencias, llámese trabajo, estudio, servicios de salud, educación, recreación, transporte, entre otros.

\subsection{Tiempos de recorrido de la población hacia los Centros de Salud ofertados en los municipios de San Pelayo y Cereté}

Los servicios de salud de este tipo existen en la mayoría de las unidades espaciales del territorio estudiado, exceptuando los corregimientos de Tres María, Pelayito y el área urbana de San Pelayo, siendo en total 20 (19 en área rural y uno en área urbana de Cereté). Como se había expresado, estos centros se caracterizan por prestar primer nivel de atención. El $65 \%$ de la población que los accede afirma hacerlo por su cercanía al lugar de residencia; sin embargo, la frecuencia de su uso no es coherente con ello, debido a que más de la mitad de los encuestados asisten a los CAMU y al Hospital con el 39\% (233 usuarios) y el 28\% (169 habitantes), respectivamente, correspondiendo el $33 \%$ (195 usuarios) a la asistencia frecuente a los centros de salud. 
Entretanto, el $35 \%$ de la población acude a los CAMU y el Hospital San Diego por la oferta permanente (diaria) del servicio de salud especializado y de mejor calidad, a diferencia de los centros de salud, lo que reitera la desigualdad e inequidad en el acceso a la salud entre la población urbana y rural. Más del $60 \%$ del área en cada municipio presenta accesibilidad física óptima con tiempos menores o iguales a 15 minutos hacia los centros de salud, cubriendo el $92 \%$ de la población de San Pelayo (39446) y el 96\% del municipio de Cereté (82092), como se observa en la Figura 3.

En su orden, la categoría de accesibilidad física favorable de 16 a 30 minutos, cubre el $20 \%$ del área municipal en 4772 personas, quienes asisten a tales infraestructuras en pocos corregimientos, como son: Tres María y Sabana Nueva, representando, en su orden, el $8 \%$ y el $1 \%$, respectivamente de la población de San Pelayo y Cereté, respectivamente.

La accesibilidad física superior a los 30 minutos entre 31 y 45 se considera desfavorable al ser mucho el tiempo que las personas emplean para llegar a un servicio de salud. En este orden, el $20 \%$ del área restante se identifica en el 3,64\% (1 636,84 ha) del área total del municipio de San Pelayo y 5,34 \% (1 482,44 ha) del área de Cereté, sin que se haya registrado población en esta categoría. De manera similar, en la categoría muy desfavorable (mayor a 46 minutos), se reconoce el 3,86\% (173,67 ha) de San Pelayo y el 5,52\% (1530,82 ha) de Cereté, en algunas de sus zonas periféricas, sobresaliendo Severá.

Cabe decir que los dos municipios estudiados están influenciados físicamente por la presencia de serranías y relieves acolinados, registrando accesibilidad física desfavorable y muy desfavorable, que dificulta el ingreso del transporte público en la parte occidental del área de estudio; así como la existencia de la planicie aluvial del río Sinú incide en lo óptimo y favorable que esta puede resultar en la parte oriental. Esta realidad se refleja en relación con la accesibilidad al resto de los equipamientos públicos de salud.
FIGURA 3.

Accesibilidad física de la población a los centros de salud municipios de San Pelayo y Cereté - Córdoba.

Fuente: Elaboración propia.
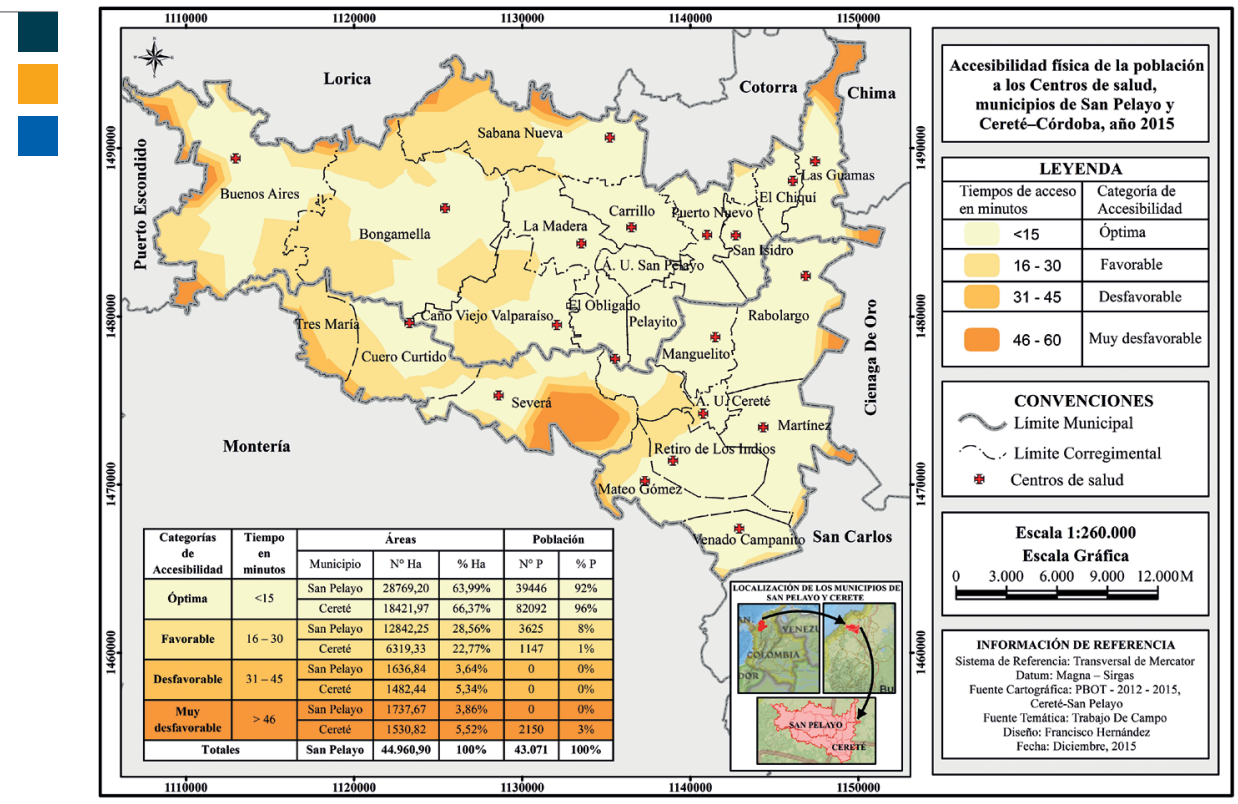


\subsection{Tiempos de recorrido de la población hacia los CAMU ofertados en los municipios de San Pelayo y Cereté}

Los CAMU prestan el primer nivel de atención como los centros de salud, disponiendo de urgencias, medicina general y especializada, sala de partos y atención diaria durante las 24 horas del día, a los que no logran llegar todos los usuarios por las distancias que les acarrea su acceso, a pesar de ser mayormente usados por los usuarios en contraste con los centros de salud.

En el área de estudio existen dos CAMU, uno por cada municipio, localizados en las cabeceras municipales cuya cobertura en la prestación de servicios es urbana y rural. Los niveles de accesibilidad física se organizan espacialmente de forma gradiente hacia las periferias (principalmente, la noroccidental), notándose que cuando más lejana se asienta la población de estos equipamientos, mayores resultan los tiempos de recorrido, por lo que estos serán desfavorables y muy desfavorables, tras superar los 31 minutos de viaje de la accesibilidad favorable.

La accesibilidad física a los CAMU en tiempos de recorrido menores o iguales a 15 minutos, que emplea la población en el municipio de San Pelayo, se observa en el 19,82\%, es decir, 8910,66 ha de su área, predominando la población urbana del $57 \%$ (24 409) y de algunos corregimientos, cercanos al CAMU, como: Carrillo, Puerto Nuevo, San Isidro, Pelayito y El Obligado, La Madera (Figura 4). Estos valores son inferiores a los encontrados a nivel de los centros de salud (Figura 3), lo que ostenta menor tamaño poblacional al compararse con el $92 \%$ indicado. Se suma el buen estado de las vías pavimentadas hasta dos carriles, existentes en sus jurisdicciones, que facilitan el acceso de las poblaciones a este tipo de equipamiento de manera más rápida.

Al contrastar San Pelayo y Cereté, presentan comportamientos similares en relación con la accesibilidad física óptima en el área urbana y rural

\section{FIGURA 4.}

Accesibilidad física de la población a los CAMU San Pelayo y El Prado, municipios de San Pelayo y Cereté - Córdoba

Fuente: Elaboración propia.

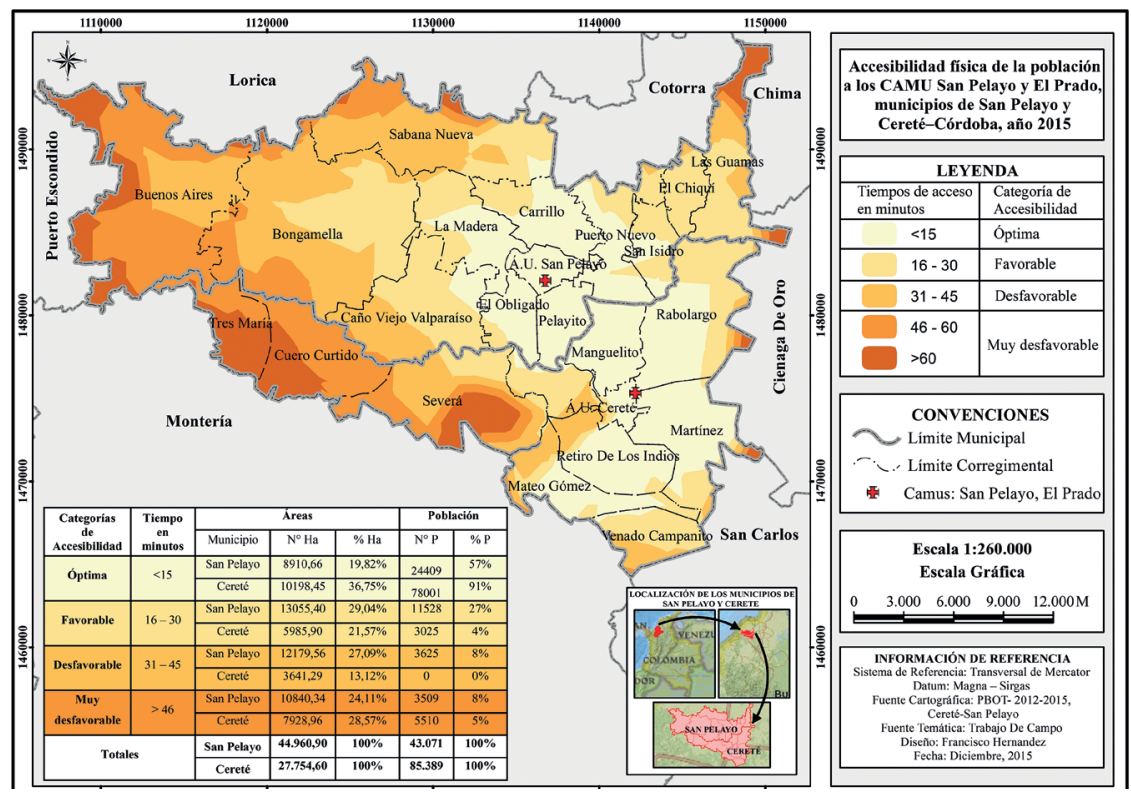


próxima al CAMU el Prado, la cual es influenciada por el buen estado vial. En el 36,75\% (10 198,45 ha) de la extensión municipal, accede el $91 \%$ de la población (78001 habitantes), 34\% más que en el municipio de San Pelayo, en los corregimientos de Mateo Gómez, Retiro de Los Indios, Martínez, Manguelito, Rabolargo. Sin embargo, al referirse a la accesibilidad a los centros de salud, la diferencia porcentual en las dos municipalidades es del $4 \%$, acudiendo en Cereté el 96\%.

Por su parte, la accesibilidad favorable (16 a 30 minutos) se halla más del $21 \%$ del área de los dos municipios (Figura 4), es así que para San Pelayo acceden el 27 \% (11528) de la población en corregimientos como Caño Viejo Valparaíso, Bongamella y en Cereté, el $4 \%$ con 3025 habitantes, en las áreas rurales El Chiquí y Las Guamas y Venado Campanito.

En la accesibilidad física desfavorable (31 a 45 minutos) está el 27,09\% (12 179,56 ha) del total del área del municipio de San Pelayo, en la que acceden el $8 \%$ (3625) de su población, perteneciente a Sabana Nueva, Bongamella, Buenos Aires, Caño Viejo Valparaíso, entre otros, mientras que para el municipio de Cereté el 13,12 \% del área (3 641,29 ha) se encuentra en esta categoría sin reportar población.

En la categoría muy desfavorable (mayor a $46 \mathrm{mi}-$ nutos), los niveles de accesibilidad física ocupan superficies de forma dispersa sin lograr continuidades espaciales entre unas y otras, como lo ilustran los anteriores mapas, reflejando en San Pelayo más del $24 \%$ del área municipal (Figura 4), $8 \%$ de su población (3509 personas) en el corregimiento de Buenos Aires (occidente del municipio), y en Cereté el $5 \%$ (5510) de los habitantes, en el $28 \%$ de la extensión, como son: Severá, Cuero Curtido y Tres María, Sabana Nueva. A medida que las poblaciones están más alejadas de las áreas urbanas donde se localizan estos equipamientos, la accesibilidad aumenta y el porcentaje de población disminuye respecto a la primera categoría de accesibilidad física.

\subsection{Tiempos de recorrido de la población hacia El Hospital Regional San Diego - municipios de San Pelayo y Cereté}

El Hospital Regional San Diego es un equipamiento de salud pública de segundo nivel de atención, localizado en la zona urbana del municipio de Cereté, con cobertura regional que sirve a poblaciones de municipios cercanos (Ciénaga de Oro, Cotorra, San Pelayo y San Carlos), resultando único en el área de estudio. El comportamiento espacial de la accesibilidad física a este hospital reporta similitud con los CAMU.

Nuevamente Cereté, en comparación con San Pelayo, registra la accesibilidad física óptima (mayores a 15 minutos), con máximo porcentaje en área del 35,87\% (9954,81 ha), correspondiente a los corregimientos de Mateo Gómez, Retiro de Los Indios (sur), Martínez (occidente), Manguelito (norte), Rabolargo (noroccidente y el área urbana). Al respecto, San Pelayo cubre en esta categoría de accesibilidad, el $6,12 \%(2750,26$ ha $)$ en la cabecera municipal y el corregimiento de Pelayito al sur del municipio. Las vías pavimentadas conectan el Hospital San Diego, lo cual posibilita su interacción entre la población que lo accede, como ocurre en Cereté con 78001 personas $(91 \%)$ y en San Pelayo para 10341 habitantes, quienes representan el 24\% (Figura 5).

En general, es de considerar que la forma ensanchada hacia el occidente del marco espacial estudiado, principalmente de la jurisdicción de San Pelayo y su territorio conformado por relieves acolinados (serranía), sumado a la distancia existente entre 
cada corregimiento en relación con la localización del Hospital San Diego en la cabecera, inciden en los niveles de accesibilidad logrados, que en este caso aluden a los favorables de 16 a 30 minutos y en adelante son desfavorables ( 31 a 45 minutos) y muy desfavorables (46 a 60 minutos y mayores a 60 ).

En este sentido, la categoría de accesibilidad favorable cubre en San Pelayo un área de 11616,43 (25,84\% del área municipal), en la cual el 35\% de la población (15282) corresponde a El Chiquí, San Isidro, Puerto Nuevo, La Madera, Carrillo (norte), El Obligado, diferenciándose de Cereté con solo el 19,55\% (5426,21 ha) del área total y en área de Venado Campanito, correspondiendo al $4 \%$ (3025 personas), quienes usan los mismos.

La accesibilidad muy desfavorable (mayores a 46 minutos) se observa en mayores áreas en San Pelayo que en Cereté, registrando el primero en 18483,10 ha (41,11\% del área de estudio), de los corregimientos de Buenos Aires y Sabana Nueva, en la cual accede un $17 \%$ de la población con 6734 habitantes, lo que se contrasta con Cereté al tener el 32,96\% (9 148,04 ha) del área, en los corregimientos de Severá, Cuero Curtido, Tres María y el $5 \%$ (4363) de la población total del municipio. Sus poblaciones se encuentran alejadas del Hospital Regional San Diego en localización periférica de los municipios, en largas distancias y nuevamente con malas condiciones viales ( $\sin$ pavimentar), que influyen en los peores niveles de accesibilidad en el área de estudio.

Al comparar la accesibilidad física a los diferentes tipos de equipamientos de salud, se destaca que la población total tiene mayor accesibilidad (óptima) a los centros de salud, donde acceden más del $90 \%$ de esta, al igual que en los CAMU en el municipio de Cereté, lo que en San Pelayo varía con solo el $57 \%$, esto debido a que en este último prevalece población rural, dispersa en el territorio, caso contrario sucede en Cereté. A su vez, la accesibilidad física hacia el Hospital Regional San Diego, por parte de la población de Cereté con porcentaje del $90 \%$, se atribuye a su condición mayormente urbana, mientras que en San Pelayo el porcentaje es menor con el $24 \%$ y rural.

\section{FIGURA 5.}

Accesibilidad física de la población al Hospital Regional San Diego, municipios de San Pelayo y Cereté - Córdoba

Fuente: Elaboración propia.

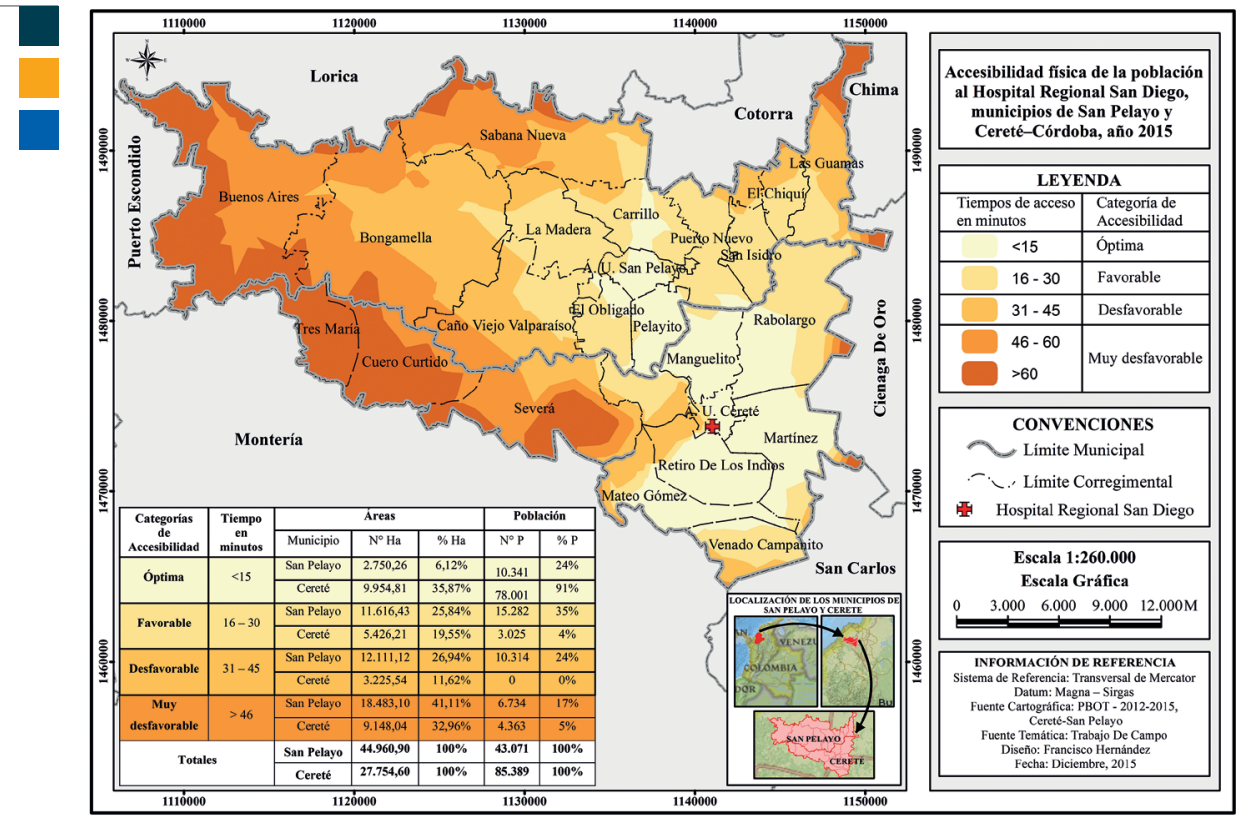


La accesibilidad favorable hacia los centros de salud en el municipio de San Pelayo es mayor a la de Cereté con el $8 \%$ y el $1 \%$ de la población que accede a los mismos, respectivamente. Esta situación sucede de forma similar en los CAMU, siendo superior el porcentaje en el primer municipio (27\%) e inferior en el segundo (4\%). La cercanía de las cabeceras corregimentales de San Pelayo al área urbana de Cereté en el cual se localiza el Hospital influye en que el $35 \%$ de la población se encuentre en esta isócrona ( 16 a 30 minutos), lo que no ocurre en Cereté pues solo lo hace el $4 \%$.

\section{Conclusiones}

El artículo que aquí se presenta se constituye en un aporte académico de referencia que será de utilidad en la toma de decisiones que llevarán a cabo las administraciones municipales y departamentales quienes participan activamente de los procesos de planificación del desarrollo socioeconómico y territorial, en busca del mejoramiento de las condiciones de accesibilidad física de la población a los equipamientos de salud. Lo antes expresado, en razón de que el estudio es la primera aproximación de diagnóstico que se tiene sobre el tema en dos localidades del departamento de Córdoba (Colombia) al año 2015, la cual ha sido medida a través de los indicadores de tiempos medios de recorrido, estado vial y tipo de vías en las diferentes unidades espaciales (área urbana y rural).

Los resultados enunciados con la accesibilidad física deberán complementarse con otros de accesibilidad socioeconómica que determine la capacidad de la población para usar tal servicio, valorando la calidad del mismo (buena o mala), teniendo en cuenta los horarios de atención, tiempo de espera para ser aten- dido y condiciones que regulan su funcionamiento (días de atención), los niveles socioeconómicos de la población, el nivel de estudios, la ocupación, el sexo y la afiliación de ella al sistema de seguridad social, para posteriormente obtener la accesibilidad geográfica.

Igualmente, se plantea el reto de generar otras investigaciones a partir del ordenamiento y la planificación territorial, sobre las actividades y servicios que se llevan a cabo en las infraestructuras de salud pública, valorando sus condiciones físico-materiales como soporte inicial para la realización de las mismas, para así continuar con la generación de pautas requeridas en la organización del territorio, sobre todo el rural, lo que serviría para trascender hacia estudios socioterritoriales y de territorialidades tanto del habitante como de la institución prestadora del servicio, producto de las acciones sociales, que se ven influenciadas por el sistema político y económico que opera en el país.

El caso de estudio aplicado en el artículo revela que la población accede a los equipamientos de salud a través del medio de transporte informal de la motocicleta $(80 \%$ de las personas encuestadas), el cual resulta predominante con respecto a los demás medios utilizados para desplazarse por la red vial, lo que indica la necesidad de medios formales y adecuados en temas de salud, siendo en su mayoría $(93,7 \%$ ) la red vial sin pavimentar, razón por la cual se convierten en un obstáculo para transitar en época de lluvias, reflejando niveles de accesibilidad desfavorable y muy desfavorable. Por tanto, a mediano y corto plazo se requiere de la mejora del 21,8\% (190 km) correspondientes a la clasificación vial sin pavimentar en los municipios de San Pelayo y Cereté, teniendo en cuenta que con esto se lograrán disminuir los tiempos de recorrido 
empleados desde los lugares de residencia hasta los equipamientos de salud.

Del mismo modo, los resultados de accesibilidad física muestran que más del $90 \%$ de las poblaciones de ambos municipios presentan la categoría óptima y favorable hacia los equipamientos como Centros de Salud, CAMU y Hospital Regional San Diego (único equipamiento de segundo nivel de atención), a excepción del $41 \%$ de San Pelayo, que registra accesibilidad desfavorable y muy desfavorable, en cuanto al último equipamiento, viéndose afectada en mayor medida la población que no dispone de una infraestructura de salud pública superior de primer nivel, de adecuadas condiciones viales y medios de transporte público-formal, localizada en algunos casos en las periferias de estas municipalidades.

Esta realidad demuestra que la población busca la satisfacción de las necesidades en salud mediante la prestación de servicios de primero y segundo nivel de atención en los centros de salud (localizados en área rural), los CAMU y el Hospital Regional de San Diego, que funcionan en la cabecera municipal, marcando diferencias en cuanto a la frecuencia de uso que hacen los habitantes, quienes, viviendo en los corregimientos y teniendo cerca a los primeros, deciden desplazarse a los segundos y al tercero, sin importar lo lejano que estén de sus lugares de residencia, toda vez que demandan los servicios ambulatorios especializados, en los primeros, como urgencias, medicina especializada en ginecología y obstetricia, sin ser quirúrgica, y en los segundos, urgencias, hospitalización, cirugía, apoyo diagnóstico y terapéutico. Es así que el $67 \%$ de la población estudiada opina frecuentar los CAMU y el Hospital Regional de San Diego (urgencias, hospitalización, cirugía, apoyo diagnóstico y terapéutico), y el 33\% los centros de salud, siendo los dos primeros tres y los últimos, veinte en los dos municipios.

De esta manera, es pertinente disponer de servicios de salud durante toda la semana en las zonas rurales y con adecuado funcionamiento, para así garantizar el acceso a la igualdad en términos de accesibilidad física hacia las infraestructuras públicas, contribuyendo al mejoramiento de la calidad de vida de la población.

Las categorías de accesibilidad física desfavorable y muy desfavorable para acceder a los servicios de salud pública coinciden con hacer parte del $22 \%$ (134 personas) de los habitantes encuestados, quienes no tienen acceso en época de lluvias, asociado esto a que la mayoría de las vías están sin pavimento y empeoran su mal estado con las mismas.

Por último, es importante resaltar el uso de los Sistemas de Información Geográfica (SIG) y con estos las herramientas que ofrecen softwares especializados para la captura, el manejo, el análisis, el modelado y el despliegue de datos espacialmente georreferenciados para la solución de problemas y obtención de resultados con alta precisión y calidad. A partir del presente estudio y demás existentes será posible evaluar la pertinencia y utilidad de estas tecnologías en la toma de decisiones, así como, comparar y analizar otras investigaciones y resultados relacionados. 


\section{Referencias}

Borroto, R., Somarribas. L. y Chamizo, H. (1994). Teoría y epistemología. Siete enfoques para el estudio geográfico de la salud humana. Revista geográfica de América Latina, 29, 11-24. Recuperado de http://www.revistas.una.ac.cr/index. php/geografica/article/viewFile/2929/2801

Congreso de la República de Colombia. (17 de febrero de 2015). Ley Estatutaria Nº 1751, por medio de la cual se regula el derecho fundamental a la salud y se dictan otras disposiciones. [Ley N. ${ }^{\circ} 1751$ de 2015].

Congreso de la República de Colombia. (1991). Constitución Política de Colombia. Artículo 49. Mediante el cual se regula el derecho fundamental a la salud y se dictan otras disposiciones.

Escalona, A. y Díez. C. (2002). Servicios básicos y calidad de vida en el espacio rural aragonés: la accesibilidad geográfica a los servicios de salud en la provincia de Teruel, Zaragoza. Centro de Estudios sobre la Despoblación y el Desarrollo Rural, Tomos 1 y 2 (inédito).

Escalona, A. y Diez. C. (2003). Accesibilidad geográfica de la población rural a los servicios básicos de salud: estudio en la provincia de Teruel. Revista de estudios sobre despoblación y desarrollo rural, 3, 111-149. Recuperado de http://www. redalyc.org/html/296/29600304/

Fuenzalida, M. (2010). Análisis de desigualdades territoriales en la oferta de equipamientos públicos: el caso de los hospitales en la red asistencial del sistema público de salud en Chile. Geografía y Sistemas de Información Geográfica (GEOSIG), 2(2), 111-125. Recuperado de https://www.academia. edu/1484055/An\%C3\%A1lisis_de_desigualdades_territoriales_en_la_oferta_ de_equipamientos_p\%C3\%BAblicos_el_caso_de_los_hospitales_en_la_red_ asistencial_del_sistema_p\%C3\%BAblico_de_salud_en_Chile

Gutiérrez, J. y García, J. (2002). Accesibilidad peatonal a la red sanitaria de asistencia primaria en Madrid. Anales de geografía de la Universidad Complutense, 269280. Recuperado de https://www.researchgate.net/publication/39283078_ Accesibilidad_peatonal_a_la_red_sanitaria_de_asistencia_primaria_en_Madrid

Huerta, U. y Källestål, C. (2012). Geographical accessibility and spatial coverage modeling of the primary health care network in the Western Province of Rwanda. International Journal Of Health Geographics, 11-40. Recuperado de https://doi. org/10.1186/1476-072X-11-40

Instituto Geográfico Agustín Codazzi (IGAC). (2009). Mapa Departamental de Córdoba

Ministerio de Transporte de la República de Colombia. (6 de agosto 2002). Ley 769. por la cual se expide el Código Nacional de Tránsito Terrestre y se dictan otras disposiciones. [Ley 769 de 2002].

Ministerio de Transporte de la República de Colombia. (20 de abril de 2010). Resolución 1384 de 2010 por la cual se adopta el método para establecer los límites de velocidad de las carreteras nacionales, departamentales, distritales y municipales de Colombia. [Resolución 1384 de 2010]. 
Ministerio de Salud de la República de Colombia. (Agosto 5 de 1994). Resolución N. ${ }^{\circ}$ 5261. Por la cual se establece el manual de actividades, intervenciones y procedimientos del plan obligatorio de salud en el sistema general de seguridad social en salud. [Resolución N. ${ }^{\circ} 5261$ de 1994]

Pérez, G. (2013). Accesibilidad geográfica y equidad en la prestación del servicio de salud: un estudio de caso para Barranquilla. Borradores de Economía, 770, 1-31. Recuperado de http://www.banrep.gov.co/sites/default/files/publicaciones/ archivos/be_770.pdf

Ramírez, L. (2009). Modelado espacial de la accesibilidad de la población a los centros de salud en el Área Metropolitana del Gran Resistencia-Chaco. Recuperado de http://hum.unne.edu.ar/investigacion/geografia/labtig/publicaciones/public34.pdf

Rodríguez, V. (2010). Medición de la accesibilidad geográfica de la población a la red de hospitales de alta resolución de Andalucía mediante sistemas de información geográfica. Geofocus, 11, 265-292. Recuperado de http://geofocus.rediris. es/2011/Articulo12_2011.pdf

Sistema de identificación de potenciales beneficiarios de programas sociales (SISBEN). (2015). Número de personas por corregimiento y área urbana municipio de San Pelayo, Córdoba-Colombia.

Sistema de identificación de potenciales beneficiarios de programas sociales (SISBEN). (2015). Número de personas por corregimiento y área urbana municipio de Cereté, Córdoba-Colombia.

Villanueba, A. (2010). Accesibilidad geográfica a los sistemas de salud y educación. Análisis espacial de las localidades de Necochea y Quequén. Revista Transporte y Territorio, 2, 136-157. Recuperado de http://www.redalyc.org/ html/3330/333027080005/ 